David J McKnight MD FRCPC

Department of Anaesthesia, University of Toronto, George C Webster, D. Min, Clinical Ethicist, St. Boniface General Hospital, Winnipeg, Manitoba

\section{Refusal of treatment and moral compromise}

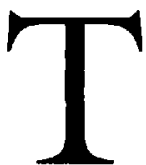

HE case report by Defalque et al. LMA in Severe Cervical Ankylosing Spondylitis, raises a difficult and increasingly common ethical problem: what is the physician to do when the patient refuses the recommended or usual treatment for a problem? A 61-year-old man with severe ankylosing spondylitis and emphysema required anaesthesia for reduction of a dislocated hip prosthesis. Two months before, for the hip replacement surgery, he had undergone what he recalled as a very unpleasant awake fibreoptic intubation. Following surgery, extubation was unsuccessful and he had a $\mathbf{1 5}$ day ICU admission of which he also had unpleasant memories. For this procedure he adamantly refused tracheal intubation.

What is the physician to do when the patient places restrictions on what is generally held to be the optimum management? In light of principles such as respect for persons, a competent patient has the right to refuse recommended treatment, even if that means harm or death. (Perhaps, from the physician's point of view, this may be seen as the right to be wrong.) But what are we to do when the patient accepts some but not all treatment? What would be the moral (not to mention the legal) responsibility of the physician if there are complications or a poor result from the modified management?

In discussing DNR orders in the operating room, Craig has pointed out the importance of providing the patient with sufficient information to make an adequately informed decision. ${ }^{1}$ In this situation, the quality of communication (not the quantity of information) between the anaesthetist and patient is of critical importance. This 'communication' goes to the very heart of the 'therapeutic relationship.' If the patient required surgery but would only consent if his trachea was not intubated, the implications of this for him and the anaesthetist (and surgeon for that matter) would have to be fully explored before the surgery. This should include an in-depth discussion of the patient's earlier experiences with intubation, current fears and/or anxieties in the face of the proposed surgery and the patient's view or understanding of his future with and without surgery. This conversation should include a discussion of the current standard of practice in anaesthe- sia and surgery, including risks and benefits for this patient with standard intubation, possible alternatives including specific risks and benefits for this patient and any difficulties or 'dis-ease' that either party might be experiencing in considering a 'no intubation scenario.'

The importance of attending to what might be called moral 'dis-ease' should not be trivialized or underestimated in this scenario. From the patient's perspective, the thought of intubation might be so overwhelming that he would choose to forgo surgery if it could only be carried out in this manner. In this sense, the proposed intervention might simply be too burdensome for him. A decision to forgo the surgical intervention, would likely create another set of complications and problems for the patient but these might be acceptable to him given his strong feelings about intubation. It is important for the anaesthetist to explore the nature of this man's fears and not simply accept his rejection of intubation at face value. In a similar way, we ought not to assume that the patient lacks decision making capacity (i.e., is incompetent) simply because he disagrees with our 'standard way of doing things.' The type of discussion envisaged here serves as an important reminder to us all that the patient is the primary decision maker in matters of treatment and care. The patient bears the burdens and consequences of these decisions and, for this reason, the patient's interests must always be at the centre of our deliberations.

The patient may be the 'primary decision maker' but, from the anaesthetist's perspective, attending to this patient without a standard intubation might simply be an unacceptable request. Here, it is important to note several things. First, the patient's request may compromise accepted standards of practice and place the patient and anaesthetist in a dangerous or unsafe situation. It might be argued that the anaesthetist should be prepared to go along with this if the patient is well informed and agrees to these risks. The anaesthetist, though, is not simply a technician nor obliged to carry out or participate in practices that are, from the anaesthetist's perspective, unsafe or unethical. This is only to say that the anaesthetist is a moral agent. This does not mean that the anaesthetist simply imposes his or her conception of 'the good' on patients. (i.e., "This is 'medically indicated' therefore 
this is what we're doing!") Rather, the anaesthetist ought to speak with the patient about any professional or ethical concerns and explore other options and possibilities (i.e., seek what Martin Benjamin calls 'an integrity preserving compromise'). ${ }^{2}$ In this context, compromise is seen to be a kind of conciliatory posture whereby both parties to a dispute find some middle ground that is acceptable to both. Here, this intermediate course would include consideration of options that might not have been initially identified by either party. This might allow both parties to hold on to deeply held or cherished beliefs and at the same time find some 'middle ground.'

In thinking about personal integrity and the problem of moral compromise, the reflective practitioner will have to explore what might be called threshold or boundary conditions. The practitioner might cooperate in some things even if he or she experiences 'dis-ease' on the grounds that others have genuinely different values and beliefs. Here, personal discernment about 'threshold or boundary' questions might push us to consider how we distinguish between a situation where we disagree with a proposed course of action but can accept it recognizing the right of a patient to make a different choice and a situation where we disagree and cannot cooperate because it would compromise our personal or professional integrity.

If we cooperate with actions or decisions that we take to be morally wrong or unethical we experience what George Webster has called 'moral residue.' This experience of 'moral residue' can have far reaching and serious consequences for the individual and the larger community. If we disagree with a patient's request but go along with it anyway we may feel that our integrity has been compromised. We know that we are troubled, we're not sure what to do about it, so we simply lower our thresholds. We rationalize our silence or acquiescence and in so doing we deceive ourselves and others. If we repeatedly compromise ourselves when confronted with serious moral questions, we can become ethically de-sensitized.

In this case report, the patient and anaesthetist were able to find an acceptable middle ground illustrating the importance of thoughtful and sensitive communication with patients. In terms of professional practice, it highlights the value of peer review in demonstrating the appropriateness of using alternative techniques in particular circumstances. Finally, the report reminds us that the 'therapeutic relationship' is not only about so-called 'clinical' or 'medical' decisions. Decisions in the 'therapeutic relationship,' as the late Paul Ramsey has observed, ${ }^{3}$ terminate in per- sons not diseases. These decisions are, by definition, moral decisions. They reveal or communicate to us some conception or understanding of 'the good' in the clinical encounter. Thoughtful and sensitive decisions in the professional-patient relationship help us to see more clearly the essential reciprocity between caregiver and patient that characterizes the healing relationship. This 'reciprocity' is ultimately about respect for persons. It doesn't mean that one party in the relationship imposes a decision on another nor does it mean that one party in the relationship must sacrifice deeply held personal or professional values and beliefs for the sake of reaching a desired outcome.

\section{Le refus du traitement et le compromis moral}

L'observation de Defalque et al. «Le masque laryngé et la spondylite cervicale avancée" (MLA in Severe Cervical Ankylosing Spondilitis) soulève un problème d'éthique difficile à résoudre et de plus en plus fréquent : quelle conduite devrait suivre un médecin quand son patient refuse un traitement reconnu et recommandé ? Un homme de 61 ans souffrant de spondylite ankylosante grave compliquée d'emphysème devait être anesthésié pour la réduction d'une luxation de prothèse de la hanche. Une intubation à l'état vigile par fibroscopie pour l'insertion de cette prothèse lui avait laissé un très mauvais souvenir. Après l'intervention, les tentatives d'extubation s'étant soldées par un échec, il avait dû séjourner pendant 15 jours à l'unité des soins intensifs, séjour dont il conservait un très mauvais souvenir. Il refusait donc catégoriquement l'intubation endotrachéale pour cette nouvelle intervention.

Que doit faire le médecin quand un patient refuse ce qui est considéré comme une prise en charge optimale ? En vertu de principes comme celui du droit au respect, le patient mentalement compétent a le pouvoir de refuser un traitement recommandé, si un préjudice ou même la mort devait en résulter (selon le point de vue du médecin, ceci constitue le droit d'avoir tort). Mais que faire quand le patient n'accepte qu'une partie du traitement mais non l'ensemble? Quelle est la responsabilité morale (oublions la responsabilité légale) du médecin si des complications ou un échec thérapeutique survenaient parce qu'il a modifié le traitement ? 
Dans son article sur l'ordre de ne pas réanimer (NPR) en salle d'opération, Craig a souligné l'importance de l'accès à une information suffisante pour permettre au patient de prendre une décision rationnelle. ${ }^{1}$ Dans cette situation, la qualité de la communication (et non celle des renseignements) entre l'anesthésiste et le patient revêt une importance capitale. Cette communication est étroitement liée à la relation thérapeutique. $\mathrm{Si}$ une intervention est nécessaire et qu'il y consent tout en refusant l'intubation, les conséquences de ce refus pour lui-même et pour l'anesthésiste (et sous cet aspect, pour le chirurgien) devraient avoir été complètement élucidées avant la chirurgie. Ceci devrait comprendre une discussion en profondeur avec le patient de l'expérience de l'intubation déjà vécue, de ses craintes et de son anxiété devant la chirurgie projetée, son opinion et sa conception de la vie avec ou sans chirurgie. Cette conversation doit inclure une discussion des normes actuelles d'exercice de l'anesthésie et de la chirurgie, incluant les risques et les bienfaits de l'intubation, les solutions de rechange possibles incluant leurs inconvénients et leurs avantages, tous les embarras ou dommages que l'une ou l'autre des personnes intéressées pourraient avoir à subir dans le cas d'un scénario où l'intubation est omise.

Sous ce scénario, l'importance de la gestion du malaise «moral» ne devrait pas être banalisée ou sousestimée. Dans la perspective du patient, l'idée de l'intubation pourrait être insupportable au point où il refuscrait l'intervention si elle ne pouvait être effectuée autrement. Pour lui, l'intervention deviendrait tout simplement un fardeau trop lourd à supporter. Son veto pourrait provoquer de nouveaux problèmes et complications en série lesquelles seraient quand même acceptables pour lui-même. Il est important pour l'anesthésiste d'explorer la nature de ses craintes et de ne pas accepter comme tel son refus de l'intubation. Nous ne devons pas présumer non plus que le patient est inapte à prendre une décision simplement parce qu'il est en désaccord avec notre façon d'agir habituelle. Le type de discussion envisagé ici doit tenir compte du fait que c'est le patient qui prend l'ultime décision en rapport avec le traitement et les soins. Le patient supporte le fardeau et les conséquences de ses décisions et, pour cette raison, ses intérêts doivent demeurer au centre de nos délibérations.

Même si c'est le patient qui décide en bout de ligne, l'anesthésiste peut juger que sa prise en charge de ce patient sans l'intubation usuelle constitue une exigence inacceptable. Ici, plusieurs commentaires s'imposent. D'abord, la demande du patient peut s'écarter des normes d'exercice en usage au point de mettre en danger à la fois le patient et l'anesthésiste. On peut toujours argumenter que l'anesthésiste devrait être prêt à procéder de cette façon si le patient est bien renseigné et s'il accepte le risque. Cependant, l'anesthésiste n'est pas un technicien et il n'a pas l'obligation d'effectuer ou de participer à des pratiques qui sont, selon lui, dangereuses et contre l'éthique. En effet, l'anesthésiste a une responsabilité morale. Ce qui ne signifie aucunement qu'il impose sa conception de ce qui est bien pour le patient (en disant par ex., "c'est un ordre médical et nous allons l'exécuter». L'anesthésiste doit plutôt expliquer à son patient ses soucis d'ordre professionnel et moral et rechercher d'autres options et d'autres solutions (c.-àd., rechercher ce que Benjamin Martin appelle «un compromis qui préserve l'intégrité»). ${ }^{2}$ Dans ce contexte, le compromis est une sorte de conciliation grâce à laquelle les deux parties à une dispute trouvent un terrain d'entente mitoyen acceptable pour les deux. Ici, ce parcours intermédiaire offre des options qui n'avaient pas été envisagées et aux intéressés la chance de sauvegarder leurs convictions profondes tout en adoptant une attitude commune.

En face du problème de l'intégrité professionnelle et du compromis moral, l'anesthésiste devra à se fixer des «balises». Il peut se sentir capable de collaborer sur certains aspects même s'il ressent un malaise dû au fait que d'autres ont des valeurs et des convictions réellement différentes. Les «balises» que nous nous serons fixées pourraient nous permettre de distinguer entre une position de désaccord avec une ligne de conduite qui reconnaît le droit au patient de faire un choix différent, et une position de désaccord qui nous interdit de collaborer parce que ce serait compromettre notre intégrité professionnelle et personnelle.

Si nous coopérons à des actes ou des décisions que nous jugeons moralement incorrects, nous éprouvons ce que George Webster appelle le «résidu moral». Cette expérience du «résidu moral» peut avoir des conséquences graves et d'une grande signification pour l'individu et pour la communauté. $\mathrm{Si}$ nous sommes en désaccord avec la demande d'un patient et que nous y cédons malgré tout, nous avons le sentiment que notre intégrité est compromise. Nous avons conscience de notre égarement, mais ne savons comment y faire face, et par conséquent, nous abaissons le seuil de notre intégrité. Nous justifions ainsi notre silence ou notre accord, et, ainsi, nous nous décevons à nos yeux et aux yeux des autres. Si nous nous discréditons à répétitions lorsque nous sommes en face de questions morales importantes, nous perdons ce qui nous reste de sens moral. 
L'observation actuelle nous montre que le patient et son anesthésiste peuvent réussir à trouver un terrain d'entente intermédiaire illustrant ainsi l'importance de la communication réfléchie et sensible. En terme d'exercice professionnel, ce compte rendu souligne la valeur du jugement des pairs en démontrant l'à-propos des techniques de rechange dans des circonstances particulières. Finalement, ce compte rendu nous rappelle que la relation thérapeutique, comme l'observait le regretté Paul Ramsey, trouve sa finalité chez des personnes et non dans des maladies. Par définition, ces décisions sont morales. Elles nous révèlent une conception ou une compréhension du «bien» dans une situation clinique. Au cours de la relation professionnel-patient, des décisions réfléchies et sensibles nous aident à voir plus clairement la réciprocité essentielle entre celui qui soigne et le patient, une caractéristique de la relation thérapeutique. Cette réciprocité concerne finalement le respect envers les personnes. Elle ne signifie pas qu'une des parties de la relation impose une décision à l'autre ni qu'un des parties doit sacrifier des valeurs personnelles, professionnelles et des convictions qui lui sont chères afin d'atteindre un résultat donné.

\section{References}

1 Craig DB. Do not resuscitate orders in the operating room. Can J Anaesth 1996; 43: 840-51.

2 Benjamin M. Splitting the Difference: Compromise and Integrity in Ethics and Politics. University Press of Kansas, 1990.

3 Ramsay P. Patient as Person. New Haven, CT, Yale University Press, 1970. 